\title{
ON THE POINTWISE DOMINATION OF A FUNCTION BY ITS MAXIMAL FUNCTION
}

\author{
J. M. ALDAZ
}

\begin{abstract}
We show that under rather general circumstances, the almost everywhere pointwise inequality $|f|(x) \leq M f(x)$ is equivalent to a weak form of the conclusion of the Lebesgue density theorem, for totally bounded closed sets. We derive both positive and negative results from this characterization.
\end{abstract}

\section{INTRODUCTION}

Let $f$ be a locally integrable function, and let $M f$ denote the centered Hardy-Littlewood maximal function of $f$. A standard proof of the almost everywhere pointwise inequality $|f|(x) \leq M f(x)$ runs as follows: Prove a weak type (1,1) inequality for $M f$, deduce the Lebesgue differentiation theorem, and use the fact that the supremum over all balls is at least as large as the limit when $r \downarrow 0$.

To a considerable extent, the usefulness of $M f$ in the presence of weak type $(1,1)$ bounds, comes from the fact that it is a.e. larger than $|f|$ but not much larger, since it is still comparable to $f$ in the $L^{p}$ sense, for $p>1$. One might suspect than when the weak type $(1,1)$ bounds fail, $M f$ should be exceedingly large, so the a.e. pointwise inequality $|f|(x) \leq M f(x)$ ought to hold. But as we shall see, this need not be the case. We show below that the inequality $|f|(x) \leq M f(x)$ a.e. is equivalent, under very general assumptions, to the following weak form of the conclusion of the Lebesgue density theorem: For all closed and totally bounded sets $F \subset \operatorname{supp} \mu$ and a.e. $x$,

$$
\limsup _{r \downarrow 0} \frac{1}{\mu(B(x, r))} \int_{B(x, r)} \mathbf{1}_{F} d \mu=\mathbf{1}_{F}(x) .
$$

It then follows from a result of D. Preiss that there exists a Gaussian measure on an infinite dimensional Hilbert space $H$ for which $|f|(x) \leq M f(x)$ fails on a set of positive measure, while by results of D. Preiss and J. Tišer the said inequality holds a.e. for some classes of Gaussian measures on $H$ (cf. [Pr], [PrTi], TTi]).

We also prove some positive results in the setting of metric measure spaces: If the space is geometrically doubling, or (without restrictions on the metric space) if the size of balls is not "too small" as the radii tend to zero, then $|f|(x) \leq M f(x)$ holds a. e., for all reasonable (meaning $\tau$-additive and finite on bounded sets) Borel measures. The last section deals with

2000 Mathematical Subject Classification. 42B25.

The author was partially supported by Grant MTM2015-65792-P of the MINECO of Spain, and also by by ICMAT Severo Ochoa project SEV-2015-0554 (MINECO). 
$\sigma$-algebras larger than the Borel sets, along the lines of [AlMi] on extensions of the Lebesgue differentiation theorem.

I am indebted to an anonymous referee for his or her very careful reading of this paper, as well as several useful suggestions.

\section{Some DEFinitions AND BACKGROUnd}

First we present the basic definitions and background material, spending some time on why the definition of metric measure spaces given below, involving $\tau$-additivity, is the right one in this context. The standard axioms for set theory, Zermelo-Fränkel with Choice, are abbreviated by ZFC. We use $B(x, r):=\{y \in X: d(x, y)<r\}$ to denote open balls, and $B^{c l}(x, r):=\{y \in X: d(x, y) \leq r\}$ to refer to metrically closed balls. It is always assumed that measures are not identically 0 .

Definition 2.1. A measure on a topological space is Borel if it is defined on the sigma algebra generated by the open sets. A Borel measure is $\tau$-smooth or $\tau$-additive, if for every collection $\left\{U_{\alpha}: \alpha \in \Lambda\right\}$ of open sets,

$$
\mu\left(\cup_{\alpha} U_{\alpha}\right)=\sup _{\mathcal{F}} \mu\left(\cup_{i=1}^{n} U_{\alpha_{i}}\right)
$$

where the supremum is taken over all finite subcollections $\mathcal{F}=\left\{U_{\alpha_{1}}, \ldots, U_{\alpha_{n}}\right\}$ of $\left\{U_{\alpha}: \alpha \in\right.$ $\Lambda\}$. We say that $(X, d, \mu)$ is a metric measure space if $\mu$ is a $\tau$-additive Borel measure on the metric space $(X, d)$, such that $\mu$ assigns finite measure to bounded Borel sets.

Recall that via the Caratheodory's outer measure construction, any measure $\mu$ on a $\sigma$ algebra can be extended in a unique way to its completion, defined on the $\sigma$-algebra of all $\mu^{*}$-measurable subsets. By a slight abuse of notation, we also call $(X, d, \mu)$ a metric measure space when the Borel measure $\mu$ is replaced by its completion, denoted again by $\mu$ (this being motivated by the fact that the equivalence classes of measurable functions in the larger $\sigma$-algebra, still have Borel representatives).

The following lemma is well known, see for instance [Bo, Theorem 7.1.7]. It is placed here as a reminder. Recall that a set $T$ is totally bounded if given any $r>0, T$ can be covered with finitely many balls of radius $r$.

Lemma 2.2. Let $(X, d)$ be a metric space endowed with a finite Borel measure $\mu$. Then every Borel set can be approximated in measure from within by closed sets. Furthermore, if $\mu$ is $\tau$-additive, then the approximating closed sets can be chosen to be totally bounded.

Proof. Call a set $A$ approximable if for every $\varepsilon>0$ there exist an open set $O$ and a closed set $C$ such that $C \subset A \subset O$ and $\mu(O \backslash C)<\varepsilon$; clearly, the closed sets $C$ are approximable (since $C=\cap_{n}\{x \in X: d(x, C)<1 / n\}$ ), the complement of an approximable set is approximable, and countable unions of approximable sets are approximable. Thus, all Borel sets are approximable. We give more detail on why countable unions of approximable sets are approximable from within. Given $\varepsilon>0$, and a sequence $\left\{A_{n}\right\}_{1 \leq n<\infty}$ of approximable sets, first reduce it, using countable additivity, to a finite sequence $\left\{A_{n}\right\}_{1 \leq n \leq N}$ satisfying $\mu \cup_{1 \leq n<\infty} A_{n}-\mu \cup_{1 \leq n \leq N} A_{n}<\varepsilon / 4$. Then select, for each $n$, a closed set $C_{n} \subset A_{n}$ such that 
$\mu\left(A_{n} \backslash C_{n}\right)<\varepsilon / 2^{n+2}$, and note that $\cup_{1 \leq n \leq N} C_{n}$ is a closed set with $\mu \cup_{1 \leq n<\infty} A_{n}-\mu \cup_{1 \leq n \leq N} C_{n}<$ $\varepsilon / 2$.

Assume next that $\mu$ is $\tau$-additive. Let $C$ be closed, and let $\varepsilon>0$. Select a finite subcollection $B_{1}^{1}, \ldots, B_{n_{1}}^{1}$ from the cover of $C$ given by $\{B(x, 1): x \in C\}$ (by $\tau$-additivity, since this cover may be uncountable) such that $\mu\left(C \backslash \cup_{1}^{N_{1}} B_{i}^{1}\right)<\varepsilon / 2$, and set $O_{1}=\cup_{1}^{N_{1}} B_{i}^{1}$. Then repeat, using succesively balls of radii $2^{-1}, 2^{-2}, \ldots$, so that at stage $k, O_{k}$ is a finite union of balls of radius $2^{-k}$, centered at points of $C$, and satisfying $\mu\left(C \backslash O_{k}\right)<\varepsilon / 2^{k}$. Then $K:=\cap_{n} \overline{O_{n}} \subset C$ is closed, totally bounded, and satisfies $\mu(C \backslash K)<\varepsilon$.

From now on, we assume that all Borel measures under consideration are finite on bounded sets. The role of $\tau$-additivity can be explained as follows: In general, uncountable unions of measurable sets need not be measurable, but when the measurable sets are open, then the uncountable unions are open, and hence Borel. Thus, $\tau$-additivity is a strengthening of countable additivity for open sets, which allows us to reduce arbitrary unions to finite unions with arbitrarily small errors.

If the metric space is separable, then uncountable unions of open sets can be reduced to countable unions, and hence $\tau$-additivity holds for all Borel measures $\mu$. Separability is sometimes assumed when defining metric measure spaces (cf. for instance [HKST, p. 62]) but this has the unfortunate consequence of excluding from the definition spaces that appear naturally in analysis, such as $L^{\infty}([0,1])$.

Also, if $X$ is not separable but $\mu$ is Radon (inner regular with respect to the compact sets) then $\mu$ is $\tau$-additive. Of course, it is natural to ask whether there are Borel measures on metric spaces that fail to be $\tau$-additive. More on this to follow soon.

Definition 2.3. Let $(X, d, \mu)$ be a metric measure space and let $g$ be a locally integrable function on $X$. For each $x \in X$, the centered Hardy-Littlewood maximal operator $M_{\mu}$ is given by

$$
M_{\mu} g(x):=\sup _{\left\{r: 0<\mu\left(B^{c l}(x, r)\right)\right\}} \frac{1}{\mu\left(B^{c l}(x, r)\right)} \int_{B^{c l}(x, r)}|g| d \mu .
$$

When the supremum is taken over radii $r \leq R$, for some fixed $R>0$, we obtain the localized maximal operator

$$
M_{\mu, R} g(x):=\sup _{\left\{r \leq R: 0<\mu\left(B^{c l}(x, r)\right)\right\}} \frac{1}{\mu\left(B^{c l}(x, r)\right)} \int_{B^{c l}(x, r)}|g| d \mu .
$$

Often we simplify notation by writing $M_{R}$ and $M$ instead of $M_{\mu, R}$ and $M_{\mu}$, when no confusion is likely to arise.

Maximal operators can be defined using open balls instead of closed balls, and this does not change their values, for we are taking suprema. The choice made here is merely for convenience, since from the viewpoint of the covering arguments given below, it is better to have closed balls. In fact, we shall utilize the definition with open balls whenever it suits us.

Recall that the complement of the support $(\operatorname{supp} \mu)^{c}:=\cup\{B(x, r): x \in X, \mu B(x, r)=0\}$ of a Borel measure $\mu$, is an open set, and hence measurable. By the convention used in 
(11), the Hardy-Littlewood maximal function $M_{\mu} g$ is well defined everywhere on $X$, even on $(\operatorname{supp} \mu)^{c}$. However, if there exists a ball $B(x, S) \subset(\operatorname{supp} \mu)^{c}$ with $R<S$, then $M_{\mu, R} g$ is not defined at $x$. Another possible convention is to leave $M_{\mu, R}$ and $M_{\mu}$ undefined off $\operatorname{supp} \mu$. Of course, which convention one uses matters little if $(\operatorname{supp} \mu)^{c}$ has measure zero. When $\mu(X \backslash \operatorname{supp} \mu)=0$ we say $\mu$ has full support.

Now $\tau$-additivity implies that $\mu$ has full support, since $X \backslash \operatorname{supp} \mu$ is a union of open balls of measure zero. Actually, the other implication also holds, for the support is always separable; otherwise, we would be able to find an uncountable collection of disjoint open balls of positive measure, contained in some larger ball (of finite measure). Thus, having full support is equivalent to $\tau$-additivity.

From the viewpoint of defining averaging (for a fixed radius) and maximal averaging operators, it is important to have both full support and balls with finite measure. Thus, metric measure spaces, as defined above, constitute a natural and sufficiently general class to deal with the type of issues addressed here.

Returning to the question whether there are Borel measures on metric spaces that fail to be $\tau$-additive, a positive answer turns out to be equivalent to the assumption of the existence of certain very large cardinals.

Definition 2.4. Let $(X, d)$ be a metric space with $d(x, y)=1$ if $x \neq y$. Suppose there exists a $0-1$ valued Borel measure on $X$, such that for all $x \in X, \mu\{x\}=0$ (and $\mu X=1$ ). Then we say that $X$ has measurable cardinality.

It is well known that the existence of measurable cardinals entails that ZFC has models, and hence by Gödel's second incompleteness theorem, measurable cardinals cannot be proven to exist within ZFC (unless ZFC is inconsistent). Thus, in standard mathematical practice we will never meet a measurable cardinal (the reader interested in obtaining additional information on these subjects, may want to consult [Bo, Theorem 1.12.44], and for a more detailed treatment, [JuWe, Chapters 12 and 23]).

If $\mathrm{ZFC}$ is consistent, then we can add as a new axiom the non-existence of measurable cardinals and obtain a new consistent theory, within which all Borel measures on a metric space are $\tau$-additive, cf. [Bo, Proposition 7.2.10]. Thus, we can consistently assume that every metric space with a Borel measure (finite on bounded sets) is actually a metric measure space as defined above.

Furthermore, if the existence of measurable cardinals is assumed, the connection between denstity results and pointwise domination by the maximal function breaks down, since it may be impossible to consider balls of small radii.

Example 2.5. Suppose $X$ is a measurable cardinal, and let $d$ be the discrete distance on $X$ $(d(x, y)=1$ if $x \neq y)$. Let $\mu$ be a $0-1$ Borel measure on $X$ that vanishes on singletons, and let $y \in X$. Now consider $\mathbf{1}_{X \backslash\{y\}}$. Define $\nu:=\mu+\delta_{y}$. With the convention from (1), the only closed balls centered at points of $X \backslash\{y\}$ we can consider have radius at least 1, and hence they contain the whole space. Thus, on $X \backslash\{y\}$ we have $M_{\nu} \mathbf{1}_{X \backslash\{y\}}=1 / 2<\mathbf{1}_{X \backslash\{y\}}$. 


\section{MAin RESUlT}

Theorem 3.1. Let $(X, d, \mu)$ be a metric measure space. The following are equivalent:

1) For all $f \in L_{l o c}^{1}(\mu), M f(x) \geq|f|(x)$ almost everywhere.

2) For all closed and totally bounded sets $F \subset \operatorname{supp} \mu$, we have

$$
\limsup _{r \downarrow 0} \frac{1}{\mu\left(B^{c l}(x, r)\right)} \int_{B^{c l}(x, r)} \mathbf{1}_{F} d \mu=\mathbf{1}_{F}(x)
$$

almost everywhere.

3) For all $f \in L_{l o c}^{1}(\mu)$ and all $R>0, M_{R} f(x) \geq|f|(x)$ almost everywhere. Furthermore, the same equivalence holds if we use open instead of closed balls.

Proof. While the present proof is written in terms of closed balls, it is easy to check that similar arguments work for open balls. Alternatively, one may notice that neither the value of the maximal function nor of the limsup are changed if we use open instead of closed balls.

First, note that 3) implies 1) always. The hypothesis of $\tau$-additivity is used as follows. By disregarding a set of measure zero if needed, we suppose that $\operatorname{supp} \mu=X$. This entails that the definition of the maximal function involves balls of all radii at every point, removing the obstacle from the preceding example.

Let us show that 1) implies 2). Suppose $F$ is closed and totally bounded, and assume 1). Then for almost every $x \in F$, we have $M \mathbf{1}_{F}(x)=\mathbf{1}_{F}(x)$. Fix one such $x$. If the supremum is attained at some fixed ball $B^{c l}(x, R)$, so

$$
\frac{1}{\mu\left(B^{c l}(x, R)\right)} \int_{B^{c l}(x, R)} \mathbf{1}_{F} d \mu=\frac{\mu\left(F \cap B^{c l}(x, R)\right)}{\mu\left(B^{c l}(x, R)\right)}=1,
$$

then $\mu\left(B^{c l}(x, R) \backslash F\right)=0$, and hence for every $0<r \leq R, \mu\left(B^{c l}(x, r) \backslash F\right)=0$. Therefore,

$$
\lim _{r \downarrow 0} \frac{\mu\left(F \cap B^{c l}(x, r)\right)}{\mu\left(B^{c l}(x, r)\right)}=1 .
$$

Suppose next that the supremum is not attained. Select a sequence of radii $\left\{r_{n}\right\}_{n \geq 1}$ such that the averages approach 1 as $n \rightarrow \infty$. By passing to a subsequence, we may assume that the radii either diverge to infinity, or tend to some $0<r_{0}<\infty$, or go to zero. Next we prove that the first two possibilities lead to contradictions.

Note first that if for some $0<r_{0}<\infty$ and some sequence $\left\{r_{n}\right\}_{n \geq 1}$ with $\lim _{n} r_{n}=r_{0}$ we have

$$
1=M \mathbf{1}_{F}(x)=\lim _{n \rightarrow \infty} \frac{1}{\mu\left(B^{c l}\left(x, r_{n}\right)\right)} \int_{B^{c l}\left(x, r_{n}\right)} \mathbf{1}_{F} d \mu,
$$

then the supremum is attained at $B^{c l}\left(x, r_{j}\right)$ for some $j \geq 0$. To see why, observe that if $r_{n} \geq r_{0}$ for infinitely many $n$, by passing to a subsequence, we may assume that $r_{n} \downarrow r_{0}$, and then

$$
\frac{1}{\mu\left(B^{c l}\left(x, r_{0}\right)\right)} \int_{B^{c l}\left(x, r_{0}\right)} \mathbf{1}_{F} d \mu=\lim _{n \rightarrow \infty} \frac{1}{\mu\left(B^{c l}\left(x, r_{n}\right)\right)} \int_{B^{c l}\left(x, r_{n}\right)} \mathbf{1}_{F} d \mu=1
$$


so we can take $j=0$. On the other hand, if $r_{n} \leq r_{0}$ for all but (perhaps) finitely many $n$ 's, we can select a subsequence, also denoted by $\left\{r_{n}\right\}_{n \geq 1}$, with $r_{n} \uparrow r_{0}$. Then we can take $j=1$, for if $\mu\left(B\left(x, r_{1}\right) \backslash F\right)>0$, the following contradiction is obtained:

$$
1=\lim _{n \rightarrow \infty} \frac{1}{\mu\left(B^{c l}\left(x, r_{n}\right)\right)} \int_{B^{c l}\left(x, r_{n}\right)} \mathbf{1}_{F} d \mu \leq 1-\frac{\mu\left(B^{c l}\left(x, r_{1}\right) \backslash F\right)}{\mu B\left(x, r_{0}\right)}<1 .
$$

Second, we show that the case $r_{n} \rightarrow \infty$ cannot happen either, so we must have

$$
1=M \mathbf{1}_{F}(x)=\limsup _{r \downarrow 0} \frac{\mu\left(F \cap B^{c l}(x, r)\right)}{\mu\left(B^{c l}(x, r)\right)},
$$

from which 2) follows, since $F$ is closed. To this end, we prove that

$$
M \mathbf{1}_{F}(x)>\limsup _{r \uparrow \infty} \frac{\mu\left(F \cap B^{c l}(x, r)\right)}{\mu\left(B^{c l}(x, r)\right)} .
$$

If $\mu X=\infty$, this follows from the fact that $\mu F<\infty$. And if $\mu X<\infty$, we select any $R>0$ and note that by hypothesis, $\mu\left(B^{c l}(x, R) \backslash F\right)>0$. Now

$$
\begin{gathered}
\limsup _{r \uparrow \infty} \frac{\mu\left(F \cap B^{c l}(x, r)\right)}{\mu\left(B^{c l}(x, r)\right)} \leq \limsup _{r \uparrow \infty} \frac{\mu\left(B^{c l}(x, r)\right)-\mu\left(B^{c l}(x, R) \backslash F\right)}{\mu\left(B^{c l}(x, r)\right)} \\
=1-\frac{\mu\left(B^{c l}(x, R) \backslash F\right)}{\mu(X)}<1 .
\end{gathered}
$$

Next, assume 2). Let $f \geq 0$ be locally integrable. To obtain 3 ) it is enough to show that for every $y \in X$ and every $R>0$, we have $M_{R} f(x) \geq f(x)$ almost everywhere on $B(y, S)$, where $S \geq R$.

Choose $\varepsilon>0$. Using Lemma 2.2, we select a simple function $0 \leq s \leq f \mathbf{1}_{B(y, 2 S)}$ of the form $s=\sum_{1}^{n} a_{i} \mathbf{1}_{F_{i}}$, where the sets $F_{i}$ are disjoint, closed and totally bounded, the coefficients $a_{i}$, strictly positive, and furthermore, $f \mathbf{1}_{B(y, 2 S)}<s+\varepsilon$, save perhaps on a subset of measure $<\varepsilon$.

We present more detail on why simple functions can be chosen in this way. First, by disjointification, a non-negative simple function $h$ can always be expressed as $h=\sum_{1}^{n} a_{i} \mathbf{1}_{D_{i}}$, where the sets $D_{i}$ are disjoint and the coefficients $a_{i}>0$. Next, given any $\delta>0$, by Lemma 2.2, for each $i \in 1, \ldots, n$ we can select $F_{i} \subset D_{i}$ with $\mu\left(D_{i} \backslash F_{i}\right)<\delta /\left(n \max \left\{a_{1}, \ldots, a_{n}\right\}\right)$, so writing $s=\sum_{1}^{n} a_{i} \mathbf{1}_{F_{i}}$, we have $\|h-s\|_{1}<\delta$, from whence it follows that this smaller class of simple functions is still dense in $L^{1}$. Finally, since $f \mathbf{1}_{B(y, 2 S)}$ is in $L^{1}$, we can find a sufficiently large constant $T$ such that $f \mathbf{1}_{B(y, 2 S)}$ and $g:=\min \left\{T, f \mathbf{1}_{B(y, 2 S)}\right\}$ are equal save perhaps on a set of measure less than $\varepsilon$. Since the latter function is bounded, non-negative and in $L^{1}$, a simple function $s$ of the prescribed form can be found so that $s \leq g<s+\varepsilon$ everywhere.

Now if $s(x)>0$, then there is a unique $i=i(x)$ such that $x \in F_{i}$. Since $\cup_{j \neq i} F_{i}$ is a finite union of closed sets, it is closed, so the distance between $x$ and $\cup_{j \neq i} F_{i}$ is strictly positive. It follows that

$$
\limsup _{r \downarrow 0} \frac{1}{\mu\left(B^{c l}(x, r)\right)} \int_{B^{c l}(x, r)} s d \mu=a_{i} \limsup _{r \downarrow 0} \frac{1}{\mu\left(B^{c l}(x, r)\right)} \int_{B^{c l}(x, r)} \mathbf{1}_{F_{i}} d \mu,
$$


so for almost all $x \in B(y, S)$ we have

$$
\begin{gathered}
M_{R} f(x) \geq M_{R} s(x) \geq \limsup _{r \downarrow 0} \frac{1}{\mu\left(B^{c l}(x, r)\right)} \int_{B^{c l}(x, r)} s d \mu \\
=\sum_{1}^{n} a_{i} \limsup _{r \downarrow 0} \frac{1}{\mu\left(B^{c l}(x, r)\right)} \int_{B^{c l}(x, r)} \mathbf{1}_{F_{i}} d \mu=\sum_{1}^{n} a_{i} \mathbf{1}_{F_{i}}(x)=s(x) .
\end{gathered}
$$

Thus, on $B(y, S)$ we have that $M_{R} f>f-\varepsilon$, save perhaps on a set of measure $<\varepsilon$, and now 3) follows by first letting $\varepsilon \downarrow 0$, and then $S \uparrow \infty$.

\section{Corollaries}

We begin with a negative consequence of the characterization presented in Theorem 3.1 .

Corollary 4.1. There is a complete metric measure space $(X, d, \mu)$ and a function $f \in L^{1}(\mu)$, such that $M f(x)<|f|(x)$ on a set of positive measure. In fact, $X$ can be taken to be an infinite dimensional separable Hilbert space, and $\mu$ a Gaussian measure on $X$.

Proof. By $[\mathrm{Pr}]$, there exists a Gaussian (probability) measure $\gamma$ on a separable Hilbert space $H$ and a Borel subset $J$ with $\gamma J<1$, such that $\gamma$-a.e. x,

$$
\lim _{r \downarrow 0} \frac{\gamma(J \cap B(x, r))}{\gamma(B(x, r))}=1 .
$$

Next select a compact $F \subset J^{c}$ with $\gamma F>0$ (recall that in complete metric spaces, compact is equivalent to closed and totally bounded). Since

$$
\gamma(J \cap B(x, r))+\gamma(F \cap B(x, r)) \leq \gamma(B(x, r)),
$$

for almost every $x$ we have

$$
\lim _{r \downarrow 0} \frac{\gamma(F \cap B(x, r))}{\gamma(B(x, r))}=0 .
$$

It follows from the proof of Theorem 3.1 that in fact we can take $f=\mathbf{1}_{F}$, where $F$ is the compact set considered in the preceding proof, so $M \mathbf{1}_{F}<\mathbf{1}_{F}$ on a set of positive measure. Actually, by considering the three possibilities studied in the said proof (the supremum is achieved when the radius tends to infinity, when it tends to zero, or for some fixed radius $r$ ) it immediately follows from Preiss' result that for every $x \in F, M \mathbf{1}_{F}(x)<1$.

We present next some positive results. Recall that the symmetric difference between two sets is defined as $A \triangle B:=(A \backslash B) \cup(B \backslash A)$. The next result is obtained via a standard adaptation of the usual arguments to the case where the lim sup is considered, instead of the $\lim$. 
Lemma 4.2. From coverings to upper densities. Let $(X, d, \mu)$ be a metric measure space. Suppose that for every closed and totally bounded set $F \subset \operatorname{supp} \mu$ and all $R, \varepsilon>0$, there exists a finite disjoint collection of balls $\left\{B^{c l}\left(x_{1}, r_{1}\right), \ldots, B^{c l}\left(x_{N}, r_{N}\right)\right\}$ such that

1) For $i=1, \ldots, N, x_{i} \in F$ and $0<r_{i}<R$;

2) $\mu\left(F \triangle \cup_{1}^{N} B^{c l}\left(x_{i}, r_{i}\right)\right)<\varepsilon$.

Then

$$
\limsup _{r \downarrow 0} \frac{1}{\mu\left(B^{c l}(x, r)\right)} \int_{B^{c l}(x, r)} \mathbf{1}_{F} d \mu=\mathbf{1}_{F}(x)
$$

almost everywhere.

Proof. Trivially, for every $x \in F^{c}$,

$$
\lim _{r \downarrow 0} \frac{\mu\left(F \cap B^{c l}(x, r)\right)}{\mu\left(B^{c l}(x, r)\right)}=0,
$$

since $F$ is closed. Thus, given $\delta \in(0,1)$, it is enough to show that

$$
\limsup _{r \downarrow 0} \frac{1}{\mu\left(B^{c l}(x, r)\right)} \int_{B^{c l}(x, r)} \mathbf{1}_{F} d \mu \geq \mathbf{1}_{F}(x)-\delta,
$$

save perhaps on a set $A \subset F$ of measure $\leq \delta$. Towards a contradiction, suppose $\mu A>\delta$ and for all $x \in A$,

$$
\limsup _{r \downarrow 0} \frac{1}{\mu\left(B^{c l}(x, r)\right)} \int_{B^{c l}(x, r)} \mathbf{1}_{F} d \mu<1-\delta .
$$

Now for each $x \in A$ there is an $r(x)>0$ such that whenever $r \leq r(x)$,

$$
\int_{B^{c l}(x, r)} \mathbf{1}_{F} d \mu<(1-\delta) \mu\left(B^{c l}(x, r)\right) .
$$

Setting $A_{n}:=\{x \in A: r(x) \geq 1 / n\}$, we have that $\mu A=\lim _{n} \mu A_{n}$, so we can choose an $L>0$ such that $\mu A_{L}>\delta$. Furthermore, by Lemma 2.2, we may suppose that $A_{L}$ is closed. Now fix $\varepsilon>0$ (with $\varepsilon \ll \delta$ ). By hypothesis, there exists a finite disjoint collection of balls $\left\{B^{c l}\left(x_{1}, r_{1}\right), \ldots, B^{c l}\left(x_{N}, r_{N}\right)\right\}$ centered at $A_{L}$, with radii bounded by $1 / L$, such that $\mu\left(A_{L} \triangle \cup_{1}^{N} B^{c l}\left(x_{i}, r_{i}\right)\right)<\varepsilon$. But now, since $A_{L} \subset F$,

$$
\begin{gathered}
\mu A_{L}=\int_{A_{L}} \mathbf{1}_{F} d \mu \leq \int_{\cup_{1}^{N} B^{c l}\left(x_{i}, r_{i}\right) \cup A_{L}} \mathbf{1}_{F} d \mu<\varepsilon+\int_{\cup_{1}^{N} B^{c l}\left(x_{i}, r_{i}\right)} \mathbf{1}_{F} d \mu \\
=\varepsilon+\sum_{1}^{N} \int_{B^{c l}\left(x_{i}, r_{i}\right)} \mathbf{1}_{F} d \mu<\varepsilon+(1-\delta) \sum_{1}^{N} \mu B^{c l}\left(x_{i}, r_{i}\right)<2 \varepsilon+(1-\delta) \mu A_{L} .
\end{gathered}
$$

Letting $\varepsilon \downarrow 0$, we obtain a contradiction.

Definition 4.3. A metric space is geometrically doubling if there exists a positive integer $D$ such that every ball of radius $r$ can be covered with no more than $D$ balls of radius $r / 2$. 
Geometrically doubling metric spaces are separable, so the following result applies to every Borel measure (finite on bounded sets) on a geometrically doubling space. We mention that beyond the case of measures that satisfy a local comparability condition (where the answer is positive, cf. [Al, Theorem 5.1]) little is known regarding the boundedness of the maximal operator for Borel measures on geometrically doubling spaces.

Corollary 4.4. Let $(X, d, \mu)$ be a geometrically doubling metric measure space. Then for all $f \in L_{l o c}^{1}(\mu), M f(x) \geq|f|(x)$ almost everywhere.

Proof. This follows from Hytönen's generalization (cf. [Hy, Lemma 3.3]) to the geometrically doubling setting, of Tolsa's Lemma on the existence of arbitrarily small doubling cubes in $\mathbb{R}^{d}$ (cf. [To, Lemma 2.8]).

By Hytönen's result, there exists a constant $C>1$ such that for $\mu$-a.e $x$ one can find a sequence of radii $r_{n}(x)$ with $\mu\left(B^{c l}\left(x, 3 r_{n}(x)\right) \leq C \mu B^{c l}\left(x, r_{n}(x)\right)\right.$ and $r_{n}(x) \downarrow 0$ as $n \rightarrow \infty$. Let $F \subset \operatorname{supp} \mu$ be a closed and totally bounded set of positive measure, and fix $\varepsilon>0$. By removing a set of measure zero if needed, we may assume that all points in $F$ are centers of decreasing sequences of small doubling balls. Choose $N \gg 1$ so that the $1 / N$-neighborhood of $F$, denoted here by $B l(F, 1 / N):=\cup_{x \in F} B(x, 1 / N)$, satisfies $\mu(B l(F, 1 / N) \backslash F)<\varepsilon / 2$. This can always be done since $F=\cap_{n \geq 1} B l(F, 1 / n)$. Using the standard argument for doubling measures we obtain a finite disjoint collection of balls $\left\{B^{c l}\left(x_{1}, r_{1}\right), \ldots, B^{c l}\left(x_{L}, r_{L}\right)\right\}$ such that for $i=1, \ldots, L, x_{i} \in F, 0<r_{i}<1 / N$, and $\mu\left(F \backslash \cup_{1}^{L} B^{c l}\left(x_{i}, r_{i}\right)\right)<\varepsilon / 2$. In this part of the proof the fact that the balls are closed is used: Given any $x \in F$ not already covered, it is always possible to find a ball $B^{c l}(x, r)$, disjoint from the balls previously selected, and with $r<1 / N$. Furthermore, since all chosen balls are contained in $B l(F, 1 / N)$, we conclude that $\mu\left(\cup_{1}^{L} B^{c l}\left(x_{i}, r_{i}\right) \backslash F\right)<\varepsilon / 2$. Now the result follows from Lemma 4.2 and Theorem 3.1 .

The next corollary assumes that the measure of balls does not decrease too fast with the radius: There are "local polynomial bounds" to such reduction.

Corollary 4.5. Let $(X, d, \mu)$ be a metric measure space. Suppose for every closed and totally bounded set $F \subset \operatorname{supp} \mu$ with $\mu F>0$, there are two functions $\psi_{F}, \phi_{F}>0$ on $F$, and a constant $c_{F}>0$, such that for all $x \in F$ we have $\phi_{F}(x) \leq 1$ and $\mu B(x, r) \geq \psi_{F}(x) r^{c_{F}}$ whenever $r \leq \phi_{F}(x)$. Then for all $f \in L_{l o c}^{1}(\mu), M f(x) \geq|f|(x)$ almost everywhere.

Proof. Select a closed and totally bounded set $F \subset \operatorname{supp} \mu$ with $\mu F>0$. To apply Lemma 4.2, it is enough to show that every point $x \in F$ is the center of arbitrarily small doubling balls. So choose a small radius $r \leq \phi_{F}(x)$ and towards a contradiction, suppose $B^{c l}(x, r)$ does not contain any ball of the form $B^{c l}\left(x, 3^{-j-1} r\right)$ satisfying $\mu B^{c l}\left(x, 3^{-j} r\right) \leq 4^{c_{F}} \mu B^{c l}\left(x, 3^{-j-1} r\right)$. Then $\mu B^{c l}(x, r)>4^{c_{F}} \mu B^{c l}\left(x, 3^{-1} r\right)>\cdots>4^{j c_{F}} \mu B^{c l}\left(x, 3^{-j} r\right) \geq(4 / 3)^{j c_{F}} r^{c_{F}} \psi_{F}(x) \uparrow \infty$ as $j \rightarrow \infty$.

Example 4.6. In the preceding corollary we make $\psi, \phi$ and $r$ depend on the closed and totally bounded set $F$, rather than taking them uniform over the whole space. This way the corollary can be applied, for instance, to spaces that are constructed putting together, say, 
manifolds of different dimensions. The role of $\phi$ is to avoid assuming hypotheses that imply fast growth of balls with large radii.

Next we give an example where a uniform $c$ does not exist. Let $e_{1, n}$ denote the first vector of the standard basis in $\mathbb{R}^{n}$, let $B^{n}\left(3 n e_{1, n}, 1\right):=\left\{x \in \mathbb{R}^{n}:\left\|x-3 n e_{1, n}\right\|_{2}<1\right\}$, and let $X$ be the disjoint union $X:=\cup_{n=6}^{\infty} B^{n}\left(3 n e_{1, n}, 1\right)$. To define a metric on $X$, within each ball $B^{n}\left(3 n e_{1, n}, 1\right)$ we use the standard euclidean distance, while if $x \in B^{n}\left(3 n e_{1, n}, 1\right)$ and $y \in B^{m}\left(3 m e_{1, m}, 1\right)$, with $n<m$, then $d(x, y)=3 m-3 n+\left\|x-3 n e_{1, n}\right\|_{2}+\left\|y-3 m e_{1, m}\right\|_{2}$. Finally, $\mu$ restricted to $B^{n}\left(3 n e_{1, n}, 1\right)$ is simply $n$-dimensional Lebesgue measure. Then there is no uniform $c$ that works over the whole space, but given $F \subset X$ compact, it can intersect at most finitely many components of $X$, so there is a ball of highest dimension, say $B^{N}\left(3 N e_{1, N}, 1\right)$, with $F \cap B^{N}\left(3 N e_{1, N}, 1\right) \neq \emptyset$. Then we can take $\phi_{F}=1$, and $\psi_{F}(x) r^{c_{F}}=V_{N} r^{N}$, where $V_{N}$ denotes the volume of the $N$-dimensional unit ball (which decreases for $n \geq 6$ ).

Finally, we mention that positive results for some classes of Gaussian measures on Hilbert spaces were already known. The following theorem is given in [Ti]:

Theorem 4.7. Let $H$ be a Hilbert space and let $\gamma$ be a Gaussian measure with the following spectral representation of its covariance operator:

$$
R x=\sum c_{i}\left(x, e_{i}\right) e_{i}
$$

where $\left(e_{i}\right)$ is an orthonormal system in $H$. Suppose $c_{i+1} \leq c_{i} i^{-\alpha}$ for a given $\alpha>5 / 2$. Then for all $f \in L^{p}(\gamma)$, where $p>1$, and almost every $x$, we have

$$
\lim _{r \downarrow 0} \frac{1}{\gamma(B(x, r))} \int_{B(x, r)}|f-f(x)| d \gamma=0 .
$$

Since $\mathbf{1}_{F}$ belongs to all $L^{p}$ spaces, we conclude that for all $f \in L^{1}(\gamma), M f(x) \geq|f|(x)$ almost everywhere. Actually, there is a previous result due to Preiss and Tišer (cf. [PrTi]) which under a weaker hypothesis (the lacunarity of the $c_{i}$, that is, there exists a $q<1$ such that for all $\left.i \geq 1, c_{i+1} \leq c_{i} q\right)$ yields the convergence in measure of the averages $\frac{1}{\gamma(B(x, r))} \int_{B(x, r)} f d \gamma$, as $r \downarrow 0$, to $f \in L^{1}(\gamma)$. Now convergence in measure entails that some subsequence converges a.e., and this is enough to ensure that $M f(x) \geq|f|(x)$ almost everywhere.

\section{The CASE OF LARGER $\sigma$-ALGEBRAS}

It is a natural follow-up question to study what happens with $\sigma$-algebras that are larger than the Borel sets. Let $(X, d, \mu)$ be a metric measure space, and let $\nu$ be an extension of $\mu$ to a $\sigma$-algebra $\mathcal{A}$ properly containing the Borel sets of $X$. Now if $L_{l o c}^{1}(\mu)=L_{l o c}^{1}(\nu)$, the characterization given in Theorem 3.1 still holds, as can be seen from the following argument. On the one hand, the class of totally bounded closed sets does not change, and on the other, given any locally integrable $\nu$-measurable function $f$, by hypothesis there is a Borel function $g$ such that $\nu$-a.e., $g=f$; since $\nu$ is an extension of $\mu$, all the $\nu$-averages of $g$ equal its $\mu$-averages, so $M_{\mu} g(x) \geq|g|(x) \mu$-a.e. $x$ iff $M_{\nu} g(x) \geq|g|(x) \nu$-a.e. $x$ iff $M_{\nu} f(x) \geq|f|(x)$ $\nu$-a.e. $x$. 
In this paper we deal with locally finite measures on metric spaces, so they are automatically $\sigma$-finite. Following [AlMi, Page 88, footnote 2], we say that $\mu$ is Borel semiregular if for every $A \in \mathcal{A}$, there is a Borel set $B$ such that $\nu(A \triangle B)=0$. Also, we say that $\mu$ is Borel regular if for every $A \in \mathcal{A}$, there exist Borel sets $B, C$ such that $B \subset A \subset C$ and $\mu(C \backslash B)=0$ (in the present context this is equivalent to AlMi, Definition 2.9.2]). Thus, Borel semiregularity is more general than Borel regularity. Under Borel semiregularity, $\mathcal{A}$-measurable simple functions have Borel representatives, so it follows that $L_{l o c}^{1}(\mu)=L_{l o c}^{1}(\nu)$ and the preceding considerations apply.

It is shown in [AlMi, Theorem 3.14] that if one starts with a doubling measure and extends it beyond the measurable sets, the Lebesgue differentiation theorem holds for the larger class of functions precisely when the extension is Borel semiregular. We mention that on geometrically doubling metric spaces, this result also holds for the class (more general than the doubling measures) of Borel measures satisfying a local comparability condition.

Without Borel semiregularity the connection between averages of measurable functions, and averages of indicator functions of closed sets, breaks down. This is probably best explained through an example. Also, it seems interesting to exhibit instances of measures that are Borel semiregular but not regular, and of measures that are not Borel semiregular.

Example 5.1. Given a metric measure space $(X, d, \mu)$, the natural, Borel regular extension of $\mu$, which we also denote by $\mu$, is defined on the $\sigma$-algebra of $\mu^{*}$-measurable sets, via the Caratheodory's construction. It can happen that in this way all sets become measurable, as is the case, for instance, when the measure is a Dirac delta, so further extensions may be impossible.

Even when such extensions are possible, moving into the realm of non-measurable sets will typically require the full force of the Axiom of Choice: By a well known result of R. Solovay, cf. [So], under the assumption that there exists an inaccessible cardinal, the ZF axioms, together with the Axiom of Dependent Choice, and the assumption that every set of reals is Lebesgue measurable, form a consistent theory.

Taking into account these considerations, let $\lambda$ be the Lebesgue measure on $X=[0,1]$, with the usual distance. Let $E \subset[0,1]$ be such that both $\lambda^{*} E=1=\lambda^{*} E^{c}$ (the existence of such sets is a well known consequence of the Axiom of Choice). Define $\lambda_{1}$ on the Borel sets of $E$ by setting, for each Borel set $B \subset[0,1], \lambda_{1} E \cap B=\lambda B$. Note that $\lambda_{1}$ is well defined: if $B_{1}$ and $B_{2}$ are Borel sets such that $E \cap B_{1}=E \cap B_{2}$, then $B_{1}$ and $B_{2}$ have the same Lebesgue measure, as can be seen from the following argument. Since $E \cap B_{1}=E \cap B_{2}=E \cap\left(B_{1} \cap B_{2}\right)$, $B_{1} \backslash\left(B_{1} \cap B_{2}\right) \subset E^{c}$, so $\lambda\left(B_{1} \backslash\left(B_{1} \cap B_{2}\right)\right)=0$. Likewise $\lambda\left(B_{2} \backslash\left(B_{1} \cap B_{2}\right)\right)=0$, so $\lambda\left(B_{1}\right)=\lambda\left(B_{1} \cap B_{2}\right)=\lambda\left(B_{2}\right)$.

Next, define $\lambda_{2}$ on the Borel sets of $E^{c}$ by setting, for each Borel set $B \subset[0,1], \lambda_{2} E^{c} \cap B=$ $\lambda B$. For every $t \in[0,1]$ set $\mu_{t}:=t \lambda_{1}+(1-t) \lambda_{2}$. Then it is easy to check that both $\mu_{0}$ and $\mu_{1}$ are Borel semiregular but not Borel regular, while for $0<t<1, \mu_{t}$ is not Borel semiregular, and the condition $M_{\mu_{t}} \mathbf{1}_{E}(x) \geq \mathbf{1}_{E}(x) \mu_{t}$-a.e. $x$ fails. In particular, for $t=1 / 2$ we have $M_{\mu_{1 / 2}} \mathbf{1}_{E} \equiv 1 / 2 \equiv M_{\mu_{1 / 2}} \mathbf{1}_{E}^{c}$. 
The situation observed in the preceding example on Lebesgue measure is typical for doubling measures, and more generally, for measures that satisfy a local comparability condition and are defined on a geometrically doubling metric space, since in this case the Vitali Covering Theorem is available (cf. [Al, Theorem 2.8]).

More precisely, let $\nu$ be a Borel measure, and let $\mu$ be an extension of $\nu$ to the $\sigma$-algebra $\mathcal{A}$, such that $\mu$ fails to be Borel semiregular. Let $E \in \mathcal{A}$ witness this failure, so for every Borel set $B, \mu(E \triangle B)>0$. By arguing as in the proof of Theorem 3.1, it is enough to show that

$$
\limsup _{r \downarrow 0} \frac{1}{\mu\left(B^{c l}(x, r)\right)} \int_{B^{c l}(x, r)} \mathbf{1}_{E} d \mu<\mathbf{1}_{E}(x)
$$

on a set of positive $\mu$-measure. Choose a decreasing sequence of open sets $O_{n}$ such that $\nu^{*}(E)=\lim _{n} \nu O_{n}$. Now by the failure of Borel semiregularity it follows that $\inf _{n} \mu\left(E^{c} \cap O_{n}\right)>$ 0 . Taking a Vitali covering of $E$ by centered closed balls inside $O_{n}$, we can select a disjoint collection $\left\{B_{i}^{c l}\right\}_{1 \leq i<L \leq \infty}$ with $\nu^{*}\left(E \backslash \cup_{1 \leq i<L} B_{i}^{c l}\right)=0$. If we had

$$
\limsup _{r \downarrow 0} \frac{1}{\mu\left(B^{c l}(x, r)\right)} \int_{B^{c l}(x, r)} \mathbf{1}_{E} d \mu=\mathbf{1}_{E}(x)
$$

a.e., we would be able to select the disjoint collection $\left\{B_{i}^{c l}\right\}_{1 \leq i<L \leq \infty}$ with $\mu\left(E^{c} \cap\left(\cup_{1 \leq i<L} B_{i}^{c l}\right)\right)$ as small as we wanted, contradicting $\inf _{n} \mu\left(E^{c} \cap O_{n}\right)>0$.

\section{REFERENCES}

[Al] Aldaz, J. M. Local comparability of measures, averaging and maximal averaging operators. To appear, Potential Analysis. Available at the Mathematics ArXiv.

[AlMi] Alvarado, Ryan; Mitrea, Marius Hardy spaces on Ahlfors-regular quasi metric spaces. A sharp theory. Lecture Notes in Mathematics, 2142. Springer, Cham, 2015.

[Bo] Bogachev, V. I. Measure Theory. (2007), Springer-Verlag.

[HKST] J. Heinonen, P. Koskela, N. Shanmugalingam, J. T Tyson, Sobolev spaces on metric measure spaces. An approach based on upper gradients. New Mathematical Monographs, 27. Cambridge University Press, Cambridge, 2015.

[Hy] Hytönen, Tuomas A framework for non-homogeneous analysis on metric spaces, and the RBMO space of Tolsa. Publ. Mat. 54 (2010), no. 2, 485-504.

[JuWe] Just, Winfried; Weese, Martin Discovering modern set theory. I and II. Graduate Studies in Mathematics, 8 and 18. American Mathematical Society, Providence, RI, 1996 and 1997.

[Pr] Preiss, D. Gaussian measures and the density theorem. Comment. Math. Univ. Carolin. 22 (1981), no. 1, 181-193.

[PrTi] Preiss, D.; Tišser, J. Differentiation of measures on Hilbert spaces. Measure theory, Oberwolfach 1981 (Oberwolfach, 1981), pp. 194-207, Lecture Notes in Math., 945, Springer, Berlin-New York, 1982.

[So] Solovay, Robert M. A model of set-theory in which every set of reals is Lebesgue measurable. Ann. of Math. (2) $9219701-56$.

[Ti] Tišer, J. Differentiation theorem for Gaussian measures on Hilbert space. Trans. Amer. Math. Soc. 308 (1988), no. 2, 655-666.

[To] Tolsa, Xavier Analytic capacity, the Cauchy transform, and non-homogeneous Calderón-Zygmund theory. Progress in Mathematics, 307. Birkhuser/Springer, Cham, 2014. 
Instituto de Ciencias Matemáticas (CSIC-UAM-UC3M-UCM) and Departamento de Matemáticas, Universidad Autónoma de Madrid, Cantoblanco 28049, Madrid, Spain.

E-mail address: jesus.munarriz@uam.es

E-mail address: jesus.munarriz@icmat.es 